IdeAs

Idées d'Amériques

$7 \mid 2016$

Cinéma et histoire dans les Amériques

\title{
Anne Deysine, La Cour Suprême des Etats-Unis. Droit, politique et démocratie
}

Paris, Dalloz, coll. « Les sens du droit », 2015, 287p.

François Vergniolle de Chantal

2 OpenEdition

Journals

Electronic version

URL: https://journals.openedition.org/ideas/1421

DOI: 10.4000/ideas. 1421

ISSN: 1950-5701

Publisher

Institut des Amériques

Electronic reference

François Vergniolle de Chantal, "Anne Deysine, La Cour Suprême des Etats-Unis. Droit, politique et démocratie", IdeAs [Online], 7| 2016, Online since 27 June 2016, connection on 20 October 2022. URL: http://journals.openedition.org/ideas/1421 ; DOI: https://doi.org/10.4000/ideas.1421

This text was automatically generated on 20 October 2022 .

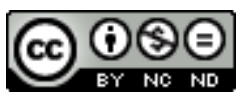

Creative Commons - Attribution-NonCommercial-NoDerivatives 4.0 International - CC BY-NC-ND 4.0 https://creativecommons.org/licenses/by-nc-nd/4.0/ 


\section{Anne Deysine, La Cour Suprême des Etats-Unis. Droit, politique et démocratie}

Paris, Dalloz, coll. « Les sens du droit », 2015, 287p.

François Vergniolle de Chantal

\section{REFERENCES}

Anne Deysine, La Cour Suprême des Etats-Unis. Droit, politique et démocratie, Paris, Dalloz, coll. « Les sens du droit », 2015, 287p.

1 Au moment où la France commence à se familiariser avec un nouveau type de contrôle de constitutionnalité depuis l'introduction de la «Question Prioritaire de Constitutionnalité » (QPC) en 2008, l'ouvrage d'Anne Deysine, juriste et professeur émérite de l'Université de Nanterre, tombe à point nommé pour expliciter les enjeux liés à ce contrôle ex post qui, aux États-Unis, est pratiqué depuis 1803. La décision historique de cette année-là créa le pouvoir moderne de "Judicial Review », donnant ainsi une ampleur inédite à l'action discrétionnaire de la Cour. Depuis lors, et comme le souligne l'ouvrage, ses pouvoirs s'étendent, de manière presque tentaculaire, à tous les aspects non seulement politiques, mais aussi, économiques et sociétaux, qui animent le débat public.

2 En effet, la Cour Suprême est non seulement l'institution centrale du Judiciaire américain en ce qu'elle est au sommet de la hiérarchie des normes (et cumule ainsi les fonctions respectives du Conseil Constitutionnel, de la Cour de Cassation et du Conseil d'État sous la $\mathrm{V}^{\mathrm{e}}$ République française), mais elle est aussi au cœur de «l'œil du cyclone » (storm center) politique, comme l'a expliqué David O’Brien dans un manuel classique. Elle est libre de se prononcer sur n'importe laquelle des questions du débat public, de l'avortement au port d'armes en passant par la réforme de l'assurancemaladie, voire sur des élections elles-mêmes, comme elle le fit en 2000 avec la décision 
Bush v. Gore. Cet "activisme ", qui a connu une intensité et des colorations différentes suivant les époques, est redevenu la nouvelle norme de la vie politique américaine depuis qu'Earl Warren, Chief Justice de 1954 à 1969, mena une "révolution des droits " dans les années soixante. Le passage de la Cour dans le camp des conservateurs à partir du milieu des années quatre-vingt ne fit que modifier l'orientation de l'activisme, sans revenir sur son intensité.

3 Le livre s'inscrit dans la tradition d'analyse pluridisciplinaire du "judicial politics", qui ne sépare pas droit et politique (p. 33), puisqu'il s'agit à la fois d'étudier « la Cour suprême en action et de l'intérieur » (p. 36), tout en évaluant sa dynamique au sein de la séparation des pouvoirs. La question globale qui anime l'ouvrage est une interrogation sur la nature politique de la Cour Suprême et son rôle dans la démocratie américaine, comme l'indique le sous-titre "droit, politique et démocratie ». Comme le dit justement $\mathrm{A}$. Deysine, le caractère « intrinsèquement politique » de la Cour ne doit pas conduire à «en déduire qu'elle est nécessairement antimajoritaire ou antidémocratique ", car ce « serait tomber dans une facilité trompeuse » (p. 37). Le plan est divisé en six chapitres. Ils abordent la place de la Cour dans le paysage constitutionnel (chapitre 1), la nomination des Justices (chapitre 2), l'autonomie du Judiciaire (chapitre 3), le processus de décision et «l'art de composer avec les contraintes » (chapitre 5); enfin, les chapitres 4 et 6 portent sur les interventions de la Cour dans les domaines politico-économiques et, plus largement, sur le rapport entre la Cour et la démocratie américaine. On a pu apprécier tout au long de ces pages la démarche didactique qui vise à clarifier le mode de fonctionnement interne de la Cour Suprême, les multiples critères qui guident les juges et les contraintes qui pèsent sur eux. Encore plus intéressant, l'auteur met également un point d'honneur à présenter les différentes philosophies interprétatives qui nourrissent les conceptions des juges, de la notion d'une "constitution vivante " (living constitution) à l'originalisme. Ce faisant, A. Deysine s'appuie sur un grand nombre de décisions bien connues en veillant pour l'essentiel à éviter les explications trop techniques et en se concentrant sur le contenu politique et juridique des textes.

4 Le lecteur est ainsi amené à comprendre les débats sur des sujets aussi variés que les innovations juridiques de la Cour Warren dans les années soixante, à commencer par la création d'un "droit à la vie privée ", nulle part mentionné dans la Constitution mais pourtant décisif pour la reconnaissance ultérieure d'un droit national à l'avortement grâce à la décision Roe v. Wade de 1973. A. Deysine revient également sur les différentes facettes de la contre-révolution conservatrice lancée par Rehnquist dès qu'il devint Chief Justice en 1986 et poursuivie par son successeur en 2005, John Roberts. Si le bilan est sans doute en retrait par rapport aux attentes des conservateurs les plus ardents, il n'en reste pas moins que la Cour conservatrice a su atteindre un certain nombre de ses objectifs. Par exemple en menant une redéfinition des relations fédérales - avec les décisions Lopez de 1995 ou Printz en 1997 - ou en accroissant la marge de manœuvre des États dans l'application du droit à l'avortement avec la décision Casey (1992). La Cour Roberts, pour sa part, a d'abord donné l'impression de s'en tenir à des interprétations étroites de la loi afin d'éviter toute confrontation sur des enjeux de substance politique avec les pouvoirs élus, comme le montra son attitude vis-à-vis de la réforme de l'assurance-maladie (National Federation of Independent Business et al. v. Sebelius, 2012). Mais cette prudence est peut-être en train de disparaitre. La Cour Roberts est la plus conservatrice au moins depuis les années trente et l'influence de la Federalist Society en son sein est à son maximum. Ainsi, Sebelius valida le "mandat» au titre du pouvoir 
fiscal du Congrès, sauvant ainsi l'essentiel de la réforme d'Obama, mais annula la disposition sur l'expansion de Medicaid en mettant en avant qu'il s'agissait d'une " coercition» des États (commandeering) et que cela allait contre le $\mathrm{X}^{\mathrm{e}}$ Amendement et les décisions New York et Printz. Le droit à l'avortement a été encadré avec Gonzales v. Carhart (2007), qui accepta le Partial-Birth Abortion Act de 2003, et demeure un enjeu des plus sensibles, comme en témoignent la décision Burwell v. Hobby Lobby Stores (2014), qui mêle autonomie des entreprises, liberté religieuse et accès à l'avortement, ou le débat en cours sur la définition des "entraves" (burden) acceptables avec le cas Whole Woman's Health v. Hellerstedt qui porte sur des restrictions adoptées au Texas en 2013. Par ailleurs, les décisions en matière de financement des campagnes (Citizens United, 2010), sur le droit individuel à porter des armes (Heller, 2008; McDonald, 2010), sur le Voting Rights Act (Shelby, 2013) sur la discrimination positive (Schuette, 2013) ou encore sur la séparation des Eglises et de l'Etat (Town of Greece, 2014), laissent peu de doute sur la poursuite d'un agenda conservateur.

5 Le rôle décisif que la Cour a ainsi pu avoir ne doit pas pour autant laisser croire qu'il existerait un quelconque « gouvernement des Juges » aux États-Unis. Si l'expression est entrée dans le vocabulaire français en 1921 grâce à l'ouvrage éponyme d'Edouard Lambert, elle est née, comme nous l'assure A. Deysine, en Amérique (p. 32). L'ouvrage souligne à de nombreuses reprises la multiplicité des contraintes qui pèsent sur le Judiciaire fédéral. Son pouvoir est en fait relativement fragile dans la mesure où sa base constitutionnelle est étroite. La Constitution de 1787 conférait à la Cour un pouvoir assez réduit, qui reposait pour l'essentiel sur les compétences énumérées à l'Article III et la "clause de suprématie " (Supremacy Clause) de l'Article VI. C'est sous l'impulsion de son premier grand Chief Justice, John Marshall (1801-1835), que la Cour a su élargir ses compétences dans les premières années de la jeune république, à un moment où un certain flou entourait encore le fonctionnement du récent texte constitutionnel. La décision Marbury v. Madison de 1803 fut une sorte de coup de force légal au terme duquel la Cour s'auto-attribua ce qui jusqu'alors était contenu en pointillés dans la clause de la suprématie, c'est-à-dire la capacité de juger de la constitutionnalité des lois.

6 Le pouvoir de la Cour est non seulement un "construit", mais il est contingent. L'ouvrage illustre abondamment les différents types de contraintes qui pèsent sur les décisions de la Cour. La Cour dépend, lors de la nomination de ses membres, des deux autres pouvoirs, la Présidence et le Sénat, transformant ainsi le choix d'un Juge en un enjeu politique de premier plan. Mais c'est aussi en son sein que la Cour est confrontée à des tensions. A. Deysine montre en particulier que les « majorités » conservatrices des dernières décennies sont fluctuantes car les oppositions y sont vives. Par ailleurs, la Cour est une institution qui est particulièrement à l'écoute de l'opinion publique. Les Juges sont grands consommateurs de sondages et, pour la plupart, ne se lancent dans un revirement de jurisprudence qu'avec circonspection. La règle cardinale du «stare decisis ", c'est-à-dire du précédent, est en effet la condition sine qua non du respect de la Cour par l'opinion et de la construction de la légitimité du Judiciaire fédéral. L'un des aspects les plus intéressants de l'ouvrage réside d'ailleurs dans son analyse de "l'affaiblissement de la règle du précédent» (p. 215-218). C'est en effet un des enjeux les plus importants de la Cour, qui, sous l'influence de la Federalist Society, grande association de juristes conservateurs, pourrait être tentée de privilégier un respect originaliste de la constitution au détriment du simple mode de décision que constitue le respect du précédent. Le potentiel quasi-révolutionnaire d'une telle démarche tient en 
haleine les analystes de la Cour Suprême et l'ouvrage d'A. Deysine permettra au lecteur, spécialiste ou simplement intéressé, d'avoir les clés de lecture nécessaires pour saisir les enjeux à venir, à commencer par le remplacement du Justice Antonin Scalia, héraut du bloc conservateur et originaliste, décédé en février dernier.

\section{AUTHORS}

\section{FRANÇOIS VERGNIOLLE DE CHANTAL}

Professeur, Paris Diderot, LARCA - UMR 8225 